\title{
Presentation Quantum Computation Based on Many Level Quantum System and SU(n) Cohered States and Qubit, Qutrit and Qubit Using Nuclear Magnetic Resonance Technique and Nuclear Quadrupole Resonance
}

\author{
YADOLLAH FARAHMAND ${ }^{1, *}{ }^{*}$ ZABIALAH HEIDARNEZHAD ${ }^{2}$, \\ FATEMEH HEIDARNEZHAD ${ }^{3}$, FATEMEH HEYDARI ${ }^{3}$ and KH. KH.MUMINOV ${ }^{1}$
}

${ }^{1 *}$ Physical Technical Institute named after S.S. Umarov, Tajikistan Academy of Sciences, Dushanbe, Tajikistan

${ }^{2}$ Young Researchers Club, Andimeshk Branch, Islamic Azad University, Andimeshk, Iran

${ }^{3}$ Department of Chemistry, Andimeshk Branch, Islamic Azad University, Andimeshk, Iran

amirfrhmnd@gmail.com

Received 25 January 2014 / Accepted 20 February 2014

\begin{abstract}
In this article we have considered the effect of a magnetic field, the Zeeman effect, nuclear magnetic resonance (NMR) and nuclear quadrupole resonance (NQR) on nuclei with spin $S$ $\geq 1 / 2$ looked. Then energy levels, transition frequencies and the energy spectra for the nuclear spins have specified. After using the group definition $\mathrm{SU}(2), \mathrm{SU}(3), \mathrm{SU}(4), \mathrm{SU}(\mathrm{N})$ OR $\mathrm{SU}(2 \mathrm{~S}+1)$, In quantum mechanics and quantum computation the range nuclear energy levels sin to one qubit, qutrit and qudit assign. We have defined the quantum states.
\end{abstract}

Keywords: Quantum theory, NMR, NQR, Spin Nuclear, SU(n), Pauli Matrices, Gell Mann matrices, Qubit, Qutrit and qudit

\section{Introduction}

Nuclear quadrupole resonance (NQR) uses radio-frequency (RF) magnetic fields to induce and detect transitions between sublevels of a nuclear ground state, a description that also applies to nuclear magnetic resonance (NMR). NMR refers to the situation where the sublevel energy splitting is predominantly due to a nuclear interaction with an applied static magnetic field, while NQR refers to the case where the predominant splitting is due to an interaction with electric field gradients within the material. So-called "pure NQR" refers to the common case when there is no static magnetic field at all. The beginning of NQR in solids dates back to the beginnings of NMR in the late 1940s and early $1950 \mathrm{~s}^{1}$. The first NQR measurements reported for a solid were by Dehmelt and Kruger using signals from $35 \mathrm{Cl}$ in transdichloroethylene ${ }^{2}$. An excellent early summary of NQR theory and technique can be found in the 1958 book by Das and $\mathrm{Hahn}^{3}$. Several more recent summaries can be 
found listed at the end of this chapter. Due to practical limitations, discussed below, NQR has not grown to be nearly as common as NMR, and is usually considered a tool for the specialist.

As is the case for NMR spectroscopy, the primary goal for NQR spectroscopy is to determine nuclear transition frequencies (i.e., energies) and/or relaxation times and then to relate those to a property of a material being studied. That property may simply be the sample temperature, for use as an NQR thermometer ${ }^{4,5}$, or even whether or not a sample is present when NQR is used for materials detection ${ }^{6}$.

On the other hand, NQR is also used to obtain detailed information on crystal symmetries and bonding, on changes in lattice constants with pressure, about phase transitions in solids, and other properties of materials of interest to solid state physicists and chemists.

As will be seen in more detail below, in order to use NQR spectroscopy one must have available an isotope with a nuclear spin I $>1 / 2$, which has a reasonably high isotopic abundance and which is at a site in a solid that has symmetry lower than tetragonal. The most common NMR isotopes, $1 \mathrm{H}, 13 \mathrm{C}$, and $15 \mathrm{~N}$ cannot be used since they have a nuclear spin $1 / 2$.

\section{Nuclear quadrupole energy levels - Classical treatment ${ }^{7}$}

Consider a nucleus of spin $1 \geq 1$ surrounded by an asymmetric electric field which, for simplicity, has axial symmetry; with two Cartesian coordinate systems, $I: \mathrm{x}$ y $\mathrm{z}$ fixed in space, and $x^{\prime} y^{\prime} z^{\prime}$ fixed on the nucleus. The $z^{\prime}$ axis is the nuclear spin axis and the $y$ and $y^{\prime}$ axes are coincident. The origin of both coordinate systems is at the center of mass of the nucleus.

The energy of interaction, E, between the nucleus and the external electric field is the product of the charge of the nucleus and V, the potential due to the field. Since the nucleus cannot be treated as a point charge, the energy term must be integrated over the nuclear volume.

$$
E=\int \rho_{N} V d v
$$

Where $\rho_{\mathrm{N}}$ is the charge density of the nucleus and is a function of $d v$, a volume element in the nucleus. The potential can be expanded a power series about the origin, thus:

$$
V=V_{0}+\sum_{i} x_{i}^{\prime} \frac{\partial V_{0}}{\partial x_{i}^{t}}+\sum_{i=1} x_{i}^{\prime} x_{i}^{\prime} \frac{\partial^{2} V_{\theta}}{\partial x_{i}^{t} \partial x_{i}^{f}}+\frac{1}{2} \sum_{i} x_{i}^{\prime 2} \frac{\partial^{2} V_{0}}{\partial^{2} x_{i}^{t}}
$$

Whence,

$$
E_{m}=\frac{1}{4} \frac{e Q V_{z z}}{I(2 I-1)}\left[3 m^{2}-I(I+1)\right]
$$

The above derivation, based on the treatment by Dehmelt ${ }^{8}$, gives a good physica1 interpretation of the energy levels. However, a more generalized form of the Hamiltonian is necessary to include, for example, the effect of axial asymmetry of the electric field. Such a Hamiltonian will now be derived quantum mechanically.

\section{Quantum mechanical derivation of the quadrupole Hamiltonian}

The Hamiltonian for the interaction between the nuclear quadrupole moment and the field gradient caused by charges totally external to the nucleus is given by the product of two tensors; $\hat{Q}$, the quadrupo $1 \mathrm{e}^{9,10}$. Moment tensor, and $\hat{V}$, the electric field gradient tensor.

$$
\hat{H}_{Q}=-\hat{Q} \cdot \hat{V}
$$

These tensors can be treated as simply third order square matrices, which have each five independent components because of symmetry. The Hamil tonian matrix components are 
derived from $\hat{Q}$ and $\hat{V}$ using the rules, of matrix multiplication. Moment tensor, and the electric field grad1ent tensor.

$$
\hat{H}_{Q}=-\sum_{m=-2}^{2} \hat{Q}^{m}-V^{\circ}{ }^{m}
$$

In this formulation of the Hamiltonian there are six non-zero components of the EFG tensor (Electric Field Gradient tensor). Without losing any generality a set of coordinates can be chosen so that all off-diagonal terms of the EFG tensor vanish. These axes are termed the principal axes of the electric field gradient. Thus it is always possible to write the Hamiltonian in terms of the principal axes as:

$$
\dot{H}_{Q}=\frac{e Q}{2 I(2 I-1)}\left[V_{x x} \dot{f}_{x}^{2}+V_{y y} \hat{f}_{y}^{2}+V_{z z} \hat{H}_{z}^{2}\right]
$$

By convent the principal axes are selected so that:

$$
\begin{gathered}
V_{x x} \leq V_{y y} \leq V_{z z} \quad \text { and } \\
\eta=\frac{v_{x x}-v_{y y}}{v_{z z}}
\end{gathered}
$$

Where, $\eta$ the asymmetry parameter, measures the deviation of the electric field from axial symmetry, along the principal $z$ axis. From equations it is apparent that $\eta$ is a unitless quantity and can vary from zero to one. It is frequently reported as a percent. From equations the Hamiltonian can be expressed as:

$$
\begin{aligned}
& \hat{H}_{Q}=\frac{e Q}{4 I(2 I-1)}\left[\left(3 f_{z}^{2}-\hat{I}^{2}\right) V_{z x}+\left(\hat{I}_{x}^{2}-\hat{f}_{y}^{2}\right)\left(V_{x x}-V_{y y}\right)\right] \\
& \hat{H}_{Q}=\frac{e Q V_{z x}}{4 I(2 I-1)}\left[\left(3 f_{z}^{2}-f^{2}\right)+\eta\left(f_{x}^{2}-f_{y}^{2}\right)\right]
\end{aligned}
$$

\section{Energy levels and transition frequencies}

In the case of axial symmetry, $\eta=0$, the pure quadrupole Hamiltonian is easily diagonalized using eigen functions of the operator $I z$ with quantum number $m=-I,-I+1, \ldots, I-1, I$. The resulting $2 I+1$ energy levels are given

$$
\text { by } E_{m}=\frac{e^{2} q Q}{4 I(2 I-1)}\left(3 m^{2}-I(I+1)\right)
$$

In this case $m$ is a good quantum number and the usual magnetic dipole transition rules apply, $\Delta m=0, \pm 1$. Defining

$$
v_{Q}=\frac{3 e^{2} q Q}{4 I(2 I-1) h}
$$

Where $h$ is Plank's constant, the allowed transition frequencies are given by

$$
v_{m . m \pm 1}=v_{Q}|(2 m \pm 1) ;| m|,| m \pm \pm 1 \mid \leq 1
$$

For the more general case of arbitrary, closed form solutions are known only for $I=1$ and $I=3 / 2$. Due to the symmetry of the Hamiltonian, all the energy levels are doubly degenerate for half-integer spin nuclei. For integer spin nuclei, of which there are very few in practice, there are an odd number of levels and the degeneracy is broken. Furthermore, since the Eigen functions of $I_{z}$ are not, in general, energy Eigen functions, additional transitions are often allowed. 


\section{Nuclear magnetic resonance}

The energy of a nuclear moment in a magnetic field, according to $\mathrm{E}=-\mu . \mathrm{B}$, is given $\mathrm{by}^{11}$

$$
E_{m I}=-\hbar y_{I} m_{I} B
$$

For nucleus of spin I, the energy of a nucleus in a magnetic field is split into $2 I+1$ Zeeman levels. A proton and other nuclei with spin $\frac{1}{2}$, have just two possible levels:

$$
E_{\mp 1 / 2}=\mp \frac{1}{2} \hbar y B
$$

With the $\alpha$-spin state $\left(m_{I}=-\frac{1}{2}\right)$ lower in energy than the $\beta$-spin state $\left(m_{I}=+\frac{1}{2}\right)$ by

$$
\Delta E=\hbar y B
$$

Figure 1 shows the energy of a proton as a function of magnetic field. In zero field $(\mathbf{B}=$ 0 ), the two spin states are degenerate. In a field $\mathbf{B}$, the energy splitting corresponds to a photon of energy $\Delta E=\hbar \omega=\hbar v$ where,

$$
\omega_{L}-\gamma B \text { or } v_{L}-\frac{\gamma B}{2 \pi}
$$

known as the Larmor frequency of the nucleus. For the proton in a field of $T, v_{L}=42: 576 \mathrm{MHz}$, as the proton spin orientation flips from $+1 / 2$ to $-1 / 2$ (Figure 1). This transition is in the radio frequency region of the electromagnetic spectrum. NMR spectroscopy consequently exploits the technology of radio wave engineering.

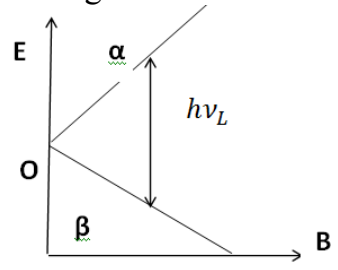

Figure 1. Energies of spin $1 / 2$ nucleus in magnetic field showing NMR transition at Larmor frequency $v_{L}$

\section{Effect of a Magnet Field - the Zeeman Effect}

When an external magnetic field, $H$, is applied to the system making an angle $\theta$ with the symmetry axis of the EFG, and azimuthal angle $\phi$ for a particular set of $\mathrm{x}$ and $\mathrm{y}$ axes, the total Hamiltonian for the system is

$$
\hat{H}=\hat{H}_{Q}+\hat{H}_{M}
$$

The quadrupole Hamiltonian is the same as that given previously in equation (26) and ${ }^{12-13}$

$$
\hat{H}_{M}=-\hbar \gamma F_{O}\left(\hat{I}_{x} \cos \theta+\hat{I}_{w} \sin \theta \cos \phi+\hat{I}_{x} \sin \theta \sin \phi\right)
$$

Whence

Energy level for $\operatorname{spin} I=1\left({ }^{2} \mathrm{H},{ }^{14} \mathrm{~N} \ldots\right)$

$$
\begin{gathered}
\hat{H}_{Q}=\frac{\omega_{Q}}{3}\left(3 \hat{I}_{\mathrm{g}}^{2}+I(I+1) \hat{I}\right) \\
\omega_{Q}=\frac{3 \pi \omega C_{Q}}{2 I(2 I-1)}
\end{gathered}
$$

There are only four known stable nuclei with integer spin: ${ }^{2} H,{ }^{6} L i$ and ${ }^{14} N$ all with $I=1$. For spin 1, the three energy levels are (Figure 2): 


$$
E_{0}=-\frac{2}{3} h v_{Q}, \quad E_{ \pm}=\frac{(1 \pm \eta)}{3} h v_{Q}
$$

and all three possible transition frequencies are allowed (Figure $3 \& 4$ ).

$$
v_{0}=\frac{2}{3} \eta v_{Q}, \quad v_{ \pm}=\left(1 \pm \frac{\eta}{3}\right) v_{Q}
$$

${ }^{1} D$ NMR spectrum of a single crystal or a liquid crystal

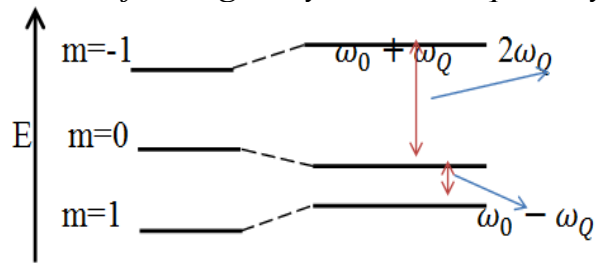

ZeemanZeeman+quadrupole $\omega$

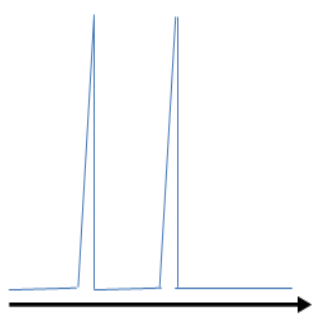

Figure 2. Energy level: Powder line shape for spin $I=1\left({ }^{2} H,{ }^{14} N \ldots\right)$

$$
\begin{array}{lll}
\eta_{\mathrm{Q}}=0 & \eta_{\mathrm{Q}}=0.2 & \eta_{\mathrm{Q}}=0.4
\end{array}
$$

(a)

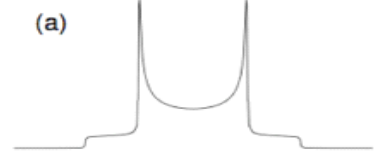

$\eta_{\mathrm{Q}}=0.6$

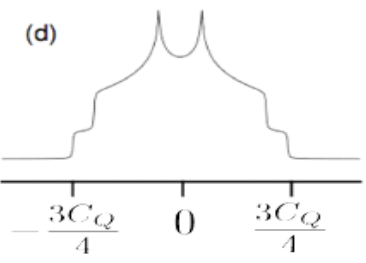

$\mathrm{kIIz}$ (b)

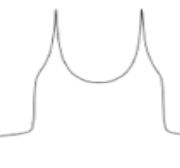

$\eta_{\mathrm{Q}}=0.8$

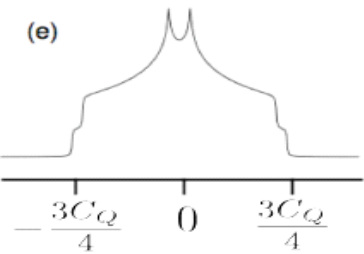

$\mathrm{kH} / \mathrm{c}$ (c)

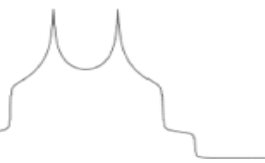

$\eta_{\mathrm{Q}}=1.0$

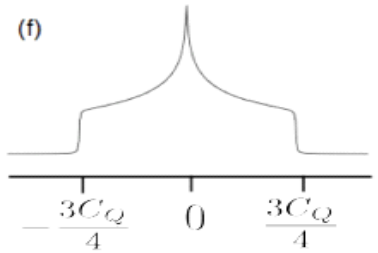

$\mathrm{kHz}$

Figure 3. The shape depends on ${ }_{l_{Q}}$ whereas the width depends on $\mathcal{C}_{\mathscr{Q}}$.

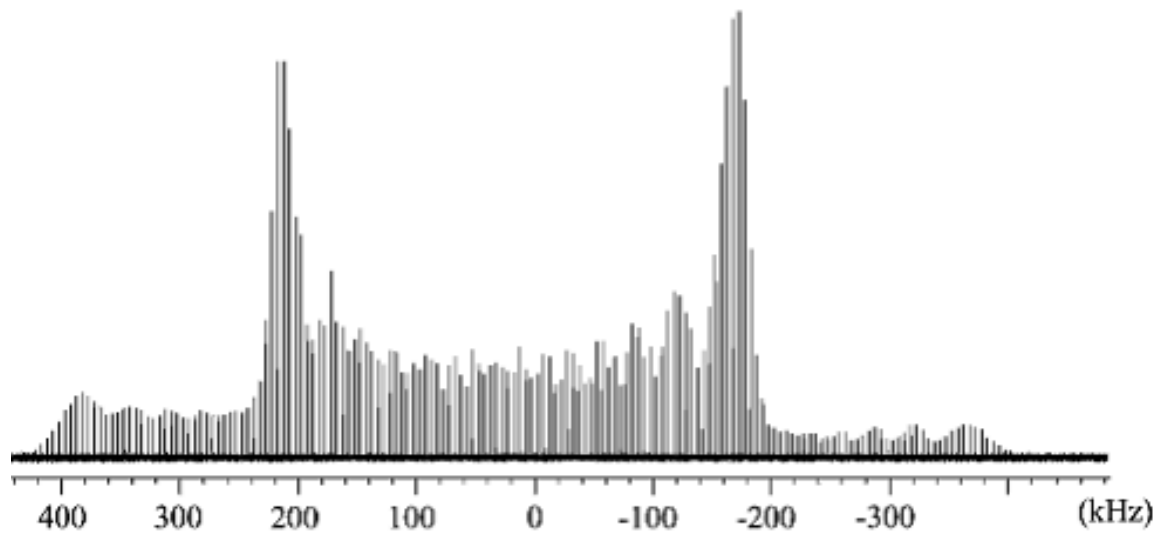

Figure 4. ${ }^{14} \mathrm{~N}$ spectrum of $\mathrm{Pb}\left(\mathrm{NO}_{3}\right)_{2}$ at $14.1 \mathrm{~T}$ and $v_{r}=6 \mathrm{kHz}$ using $16000 \mathrm{scans}^{17}$ 


\section{Spin $3 / 2$}

Much of the NQR work in the literature is for spin 3/2 nuclei, which have two doubly degenerate energy levels (Figure 5),

$$
E_{+\frac{3}{2}}-\hbar v_{Q}\left(1+\frac{\eta_{i}^{2}}{3}\right)^{\frac{1}{2}}, \quad E_{+\frac{1}{2}}=-\hbar v_{Q}\left(1+\frac{\eta^{2}}{3}\right)^{\frac{1}{2}}
$$

and hence only one (non-zero frequency) transition,

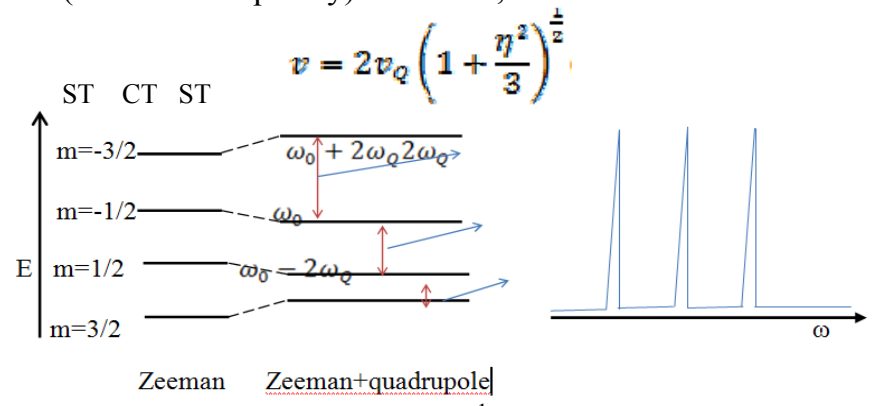

Other half-integer spins

Figure 5. Energy level ${ }^{1} \mathrm{D}$ NMR spectrum

Exact solutions are not known for $I>3 / 2$. Tabulated results can be used ${ }^{14}$ or it is now quite easy to diagonalizable the Hamiltonian numerically. Expansions valid for smaller values of $\eta$ are also available ${ }^{15,16}$. Results of numerical computations for half-integer spins 5/2,7/2, and 9/2 are shown in Figure $6 \& 7$. As is customary, the levels are labeled according to the largest component of the wave function, though $m$ is only a good quantum number when $\eta$ $=0$. In addition, when $\eta=0$ virtually all possible transitions are allowed though many are extremely weak. This is similar to what occurs for ${ }^{10} B$, mentioned above. The dotted lines in Figure $6 \& 7$ indicate some of these weaker transitions, which are not allowed at all when $\eta$ $=0$ but which may be usable for large $\eta$. Those weaker transitions are rarely used in practice but can be helpful when disentangling spectra observed for samples with multiple sites having large $\eta$. When $\eta=0$ it is possible, with some effort, to obtain exact solutions for half-integer spins up to $I=9 / 2$. The resulting energy levels for these $I$ are, in units of $h v_{Q}$.

$$
I-\frac{5}{2}: 0, \pm \sqrt{\frac{112}{9}}, I-\frac{7}{2}: \pm z\left[7 \pm 4 \sqrt{\frac{7}{3}}\right]^{\frac{1}{2}}, I-\frac{9}{2}: 0, \pm z\left[z z \pm \sqrt{\frac{748}{3}}\right]^{\frac{1}{2}}
$$

${ }^{1} D$ spectra for $\operatorname{spin} I \geq 5 / 2$ :

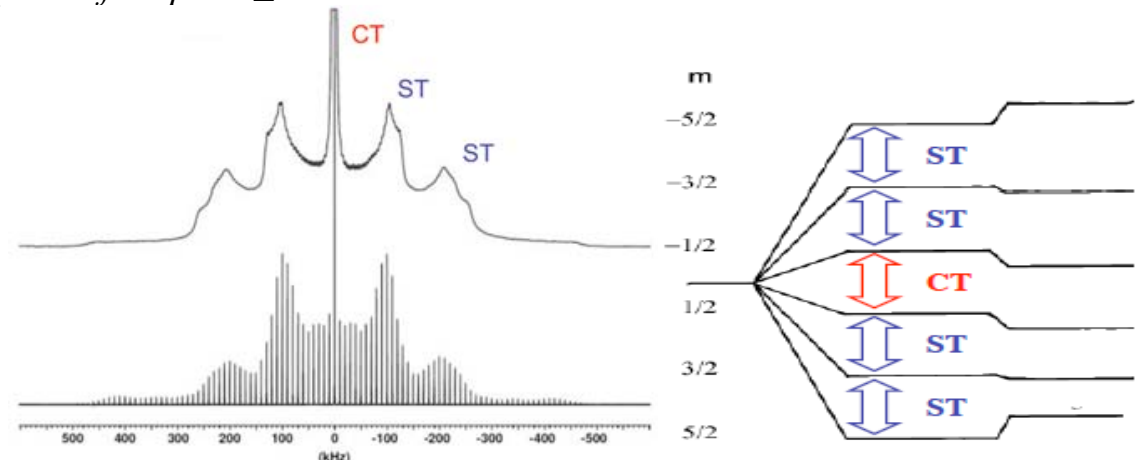

Figure 6. ${ }^{55} \mathrm{Mn}(\mathrm{I}=5 / 2){ }^{1} \mathrm{D}$ spectrum of $\mathrm{KMnO}_{4}{ }^{17} \mathrm{Zeeman}$, Zeeman+quadrupole 


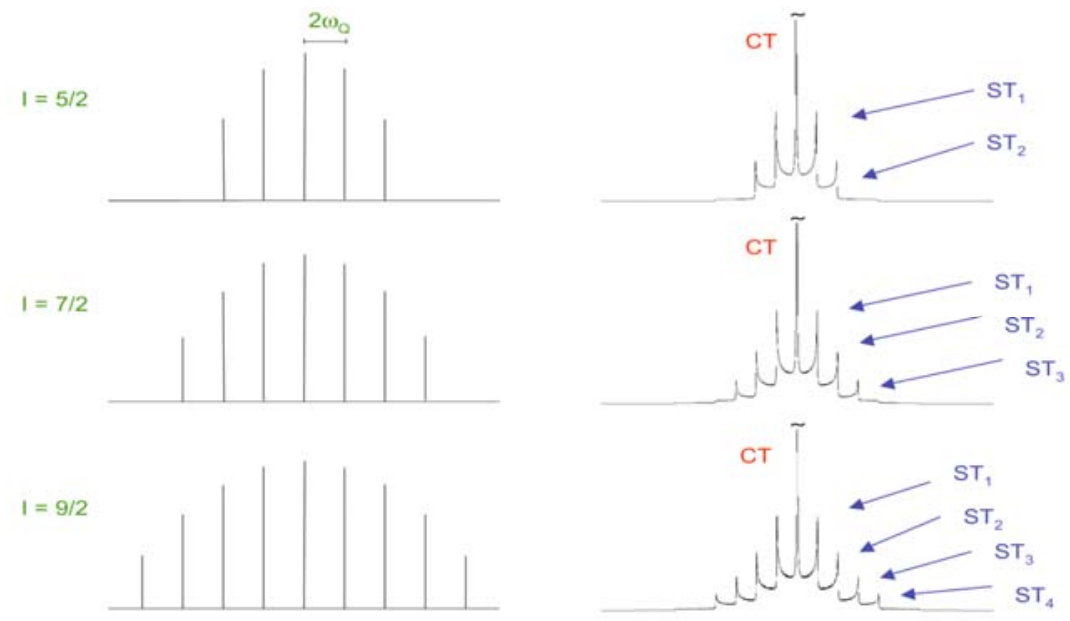

Figure 7. ${ }^{1} \mathrm{D}$ spectra for $\operatorname{spin} I \geq 5 / 2$

\section{Group Definition}

$\mathrm{SU}(2)$ is the group of all $2 \times 2$ unitary matrices with determinant 1 , elements are Complex. $\mathrm{SU}(3)$ is the group of all $3 \times 3$ unitary matrices with determinant 1 , and $\mathrm{SU}(\mathrm{n})$ is the group of all $n \times n$ unitary matrices with determinant 1 elements are complex.

Unitary matrix $\mathrm{U}, U^{\dagger} U=I$

$\mathrm{SU}(2) \operatorname{dim}=3 ; \mathrm{SU}(3) \operatorname{dim}=8$ and $\mathrm{SU}(\mathrm{n})$ groups have dimension $n^{2}-1$

\section{Qubit and SU(2) and the Pauli Matrices}

A qutbit $|\psi\rangle=\alpha_{0}|0\rangle+\alpha_{1}|1\rangle$ where, $\alpha_{0}, \alpha_{1} \in C,\left|\alpha_{0}\right|^{2}+\left|\alpha_{1}\right|^{2}=1$ is a state vector in the Hilbert space of states $H(2)$ of a 2-level system. It is spanned by an

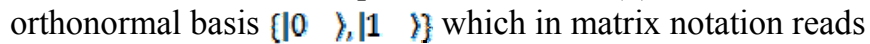

$$
|0\rangle \rightarrow\left(\begin{array}{l}
1 \\
0
\end{array}\right),|1\rangle \rightarrow\left(\begin{array}{l}
0 \\
1
\end{array}\right)
$$

The infinitesimal generators of $\mathrm{SU}(2)$ are $\sigma_{i}$ where $\sigma_{l}, \sigma_{2}$, and $\sigma_{3}$ and are the Pauli matrices. The Pauli matrices are Hermitian, that is, they have real eigen values, and traceless

$$
\sigma_{1}=\left(\begin{array}{ll}
0 & 1 \\
1 & 0
\end{array}\right), \sigma_{2}=\left(\begin{array}{cc}
0 & -t \\
i & 0
\end{array}\right), \sigma_{3}=\left(\begin{array}{cc}
1 & 0 \\
0 & -1
\end{array}\right)
$$

For a spin $1 / 2$ particle, like an electron or a proton... and ${ }^{1} \mathrm{H}_{2}{ }^{13} \mathrm{C}_{3}{ }^{15} \mathrm{~N}_{2}{ }^{31} \mathrm{P}, \ldots$ for $\mathrm{I}=\frac{\mathrm{8}}{2}$

\section{Pauli Matrices Commutation}

The Pauli matrices do not commute physically this is because one cannot simultaneously measure spin in more than one direction mathematically this is because $\mathrm{SU}(2)$ is nonAbelian. The commutation relation is:

$$
\sigma_{i} \sigma_{f}=2 i \varepsilon_{i f k} \sigma_{k}
$$

Qutrit and SU(3) and the Gell-Mann Matrices

$$
\text { A qutrit }\left|\psi k=\alpha_{0}\right| 0 \quad \xi+\alpha_{1}|1 \quad\rangle+\left.\alpha_{2}\right|_{2} \quad \xi
$$

where, $\alpha_{0}, \alpha_{1}, \alpha_{2} \in C,\left|\alpha_{0} \quad\right|^{2}+\left|\alpha_{1} \quad\right|^{2}+\left|\alpha_{2} \quad\right|^{2}=1$ is a state vector in the Hilbert space of states $H(3)$ of a 3 -level system. It is spanned by an orthonormal basis $\{|0 \quad \eta, 1 \quad\rangle, \mid 2 \quad \eta\}^{2}$ which in matrix notation reads 


$$
10\rangle \rightarrow\left(\begin{array}{l}
1 \\
0 \\
0
\end{array}\right),|1\rangle \rightarrow\left(\begin{array}{l}
0 \\
1 \\
0
\end{array}\right),|2 \quad\rangle \rightarrow\left(\begin{array}{l}
0 \\
0 \\
1
\end{array}\right)
$$

By analogy to $\mathrm{SU}(2), \mathrm{SU}(3)$ has infinitesimal generators $i \lambda i$ where $\lambda i$ are the Gell-Mann matrices. The Gell-Mann matrices are a generalization of the Pauli matrices and they have similar properties. There are eight $3 \times 3$ matrices which can be a representation of the 8 gluons that mediate. Quantum chromo dynamics (QCD), also known as the strong force. These matrices act on vectors with three elements that represent the three color charges associated with the strong force.

\section{Gell-Mann Matrices}

Where $\vec{n}$ is a real 8 -vector, and components of $\vec{\lambda}$ are the (Hermitian, traceless) Gell Mann matrices

$$
\begin{gathered}
\lambda_{1}=\left(\begin{array}{lll}
0 & 1 & 0 \\
1 & 0 & 0 \\
0 & 0 & 0
\end{array}\right), \lambda_{2}=\left(\begin{array}{ccc}
0 & -i & 0 \\
i & 0 & 0 \\
0 & 0 & 0
\end{array}\right), \lambda_{3}=\left(\begin{array}{ccc}
1 & 0 & 0 \\
0 & -1 & 0 \\
0 & 0 & 0
\end{array}\right) \lambda_{4}=\left(\begin{array}{lll}
0 & 0 & 1 \\
0 & 0 & 0 \\
1 & 0 & 0
\end{array}\right) \\
\lambda_{y}=\left(\begin{array}{ccc}
0 & 0 & -i \\
0 & 0 & 0 \\
i & 0 & 0
\end{array}\right), \lambda_{0}=\left(\begin{array}{lll}
0 & 0 & 0 \\
0 & 0 & 1 \\
0 & 1 & 0
\end{array}\right), \lambda_{y}=\left(\begin{array}{ccc}
0 & 0 & 0 \\
0 & 0 & -i \\
0 & i & 0
\end{array}\right), \lambda_{\mathrm{g}}=\frac{1}{\sqrt{3}}\left(\begin{array}{lll}
1 & 0 & 0 \\
0 & 1 & 0 \\
0 & 0 & 0
\end{array}\right)
\end{gathered}
$$

For a spin 1 particle, like: ${ }^{2} \mathrm{H}_{2}{ }^{6} \mathrm{Li}_{2}{ }^{14} \mathrm{~N} \quad \ldots$ for $\mathrm{I}=1$

Qudit and $S U(4) \ldots . S U(n)$

A qutrit $|\psi \quad\rangle=\alpha_{0}|0 \quad\rangle+\alpha_{1}|1\rangle+\alpha_{2}|2 \quad\rangle+\alpha_{3}|3 \quad\rangle$

where $\alpha_{0,} \alpha_{1}, \alpha_{2} \in C,\left|\alpha_{0} \quad\right|^{2}+\left|\alpha_{1} \quad\right|^{2}+\left|\alpha_{2} \quad\right|^{2}+\left|\alpha_{3} \quad\right|^{2}=1$ is a state vector in the Hilbert space of states $H(4)$ of a 4-level system. It is spanned by an orthonormal basis

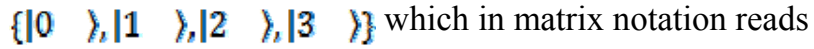

$\left.|0 \quad\rangle \rightarrow\left(\begin{array}{l}1 \\ 0 \\ 0 \\ 0\end{array}\right),|1 \quad\rangle \rightarrow\left(\begin{array}{l}0 \\ 1 \\ 0 \\ 0\end{array}\right),\left|2 \quad \gamma \rightarrow\left(\begin{array}{l}0 \\ 0 \\ 1 \\ 0\end{array}\right),\right| 3 \quad\right\rangle \rightarrow\left(\begin{array}{c}0 \\ 0 \\ 0 \\ 1\end{array}\right)$

Where $T_{i}$ are generators of SU(4) group that related to the following 15 matrices.

For a spin $3 / 2$ particle, like ${ }^{7} \mathrm{Li}_{2}{ }^{11} \mathrm{~B}_{2}{ }^{23} \mathrm{Na}_{2}{ }^{33} \mathrm{~S}_{2}{ }^{37,35} \mathrm{Cl}_{2} \ldots .$. for $\mathrm{I}=\frac{3}{2}$

A qudit $|\psi\rangle=\sum_{i=0}^{n} \alpha_{i}|i \quad\rangle$ where $\alpha_{0}, \alpha_{1}, \ldots \ldots \alpha_{n} \in C, \sum_{i=0}^{n}\left|\alpha_{0} \quad\right|^{2}=1$ is a state vector in the Hilbert space of states $H(\mathrm{n})$ of a n-level system. It is spanned by an orthonormal basis

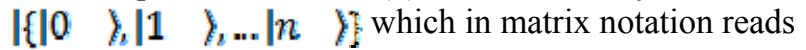

$|0\rangle-\left(\begin{array}{c}1 \\ 0 \\ 0\end{array}\right),|1\rangle-\left(\begin{array}{c}0 \\ 1 \\ 0\end{array}\right), \ldots \mid n>-\left(\begin{array}{c}0 \\ 0 \\ 1\end{array}\right)$

$|0\rangle \rightarrow$

Where $M_{i}$ are generators of $\mathrm{SU}(\mathrm{n})$ or $\mathrm{SU}(2 \mathrm{~S}+1)$ group that related to the following $(2 S+1)^{2}-1$ matrices.

For a spin $\geq 5 / 2$ particle, like:

${ }^{27} \mathrm{Al},{ }^{17} \mathrm{O},{ }^{25} \mathrm{Mg},{ }^{47} \mathrm{Ti},{ }^{55} \mathrm{Mn},{ }^{86} \mathrm{Rb}, \ldots$ for $\mathrm{I}=5 / 2$ and

${ }^{43} \mathrm{Ca},{ }^{45} \mathrm{Sc},{ }^{49} \mathrm{Ti},{ }^{55} \mathrm{Mn},{ }^{253} \mathrm{ES}$... for $I=7 / 2$ and

${ }^{87} \mathrm{Sr},{ }^{93} \mathrm{Nb}, \ldots$ for $\mathrm{I}=9 / 2$

\section{Conclusion}

The reviews of this article NMR and NQR Nuclears $S \geq 1 / 2$. This conclusion is reached. The energy spectra of these nuclei can be identified. And as it isqubit, qutrit and qudit Introduced on a quantum computer. Example qubit For a spin $1 / 2$ Nuclears, an electron or a proton...like 
${ }^{1} H,{ }^{13} \mathrm{C},{ }^{15} \mathrm{~N},{ }^{31} \mathrm{P} \ldots$ for $I=1 / 2$ and Forqutrit a spin 1 Nuclears,

Like: ${ }^{2} \mathrm{H},{ }^{6} \mathrm{Li},{ }^{14} \mathrm{~N}, \ldots$ for $I=1$ and Forqudit a spin $3 / 2$ Nuclears,

Like: ${ }^{7} \mathrm{Li},{ }^{11} \mathrm{~B},{ }^{23} \mathrm{Na},{ }^{33} \mathrm{~S},{ }^{37.35} \mathrm{Cl}, \ldots$ for $\mathrm{I}=3 / 2$ and for a spin $\geq 5 / 2$ Nuclears

Like: ${ }^{27} \mathrm{Al},{ }^{17} \mathrm{O},{ }^{25} \mathrm{Mg},{ }^{47} \mathrm{Ti},{ }^{55} \mathrm{Mn},{ }^{86} \mathrm{Rb}, \ldots$ for $\mathrm{I}=5 / 2$ and

${ }^{43} \mathrm{Ca},{ }^{45} \mathrm{Sc},{ }^{49} \mathrm{Ti},{ }^{55} \mathrm{Mn},{ }^{253} \mathrm{Es} \ldots$ for $I=7 / 2$ and

${ }^{87} \mathrm{Sr},{ }^{93} \mathrm{Nb}, \ldots$ for $\mathrm{I}=9 / 2$

\section{References}

1. Pound R V, Phys Rev., 1950, 79, 685-702.

2. Dehmelt H G and Kruger H, Naturwiss, 1950, 37(5), 111-112;

DOI:10.1007/BF00623717

3. Das T P and Hahn E L, Nuclear Quadrupole Resonance Spectroscopy, Supplement 1 of Solid State Physics, Seitz FTurnbull D, (Eds.), (New York, Academic)., 1958.

4. Ohte A, Iwaoka H, Mitsui K, Sakurai H and Inaba A, Metrologia., 1979, 15(4), 195-199.

5. Huebner M, Leib J and Eska G, J Low Temp Phys., 1999, 114(1-2), 203-230; DOI:10.1023/A:1021810105778

6. Garroway A N, Buess M L, Miller J B, Suits B H, Hibb A D, Barrall G A, Matthews R and Burnett L J, IEEE Trans Geosci Remote Sens., 2001, 39(6), 1108-1118.

7. Weber K E and Etodd J, Rev Sei Instr., 1962, 33, 390; DOI:10.1063/1.1717860

8. Dehmelt H G, Am J Phys., 1954, 22, 110; DOI:10.1119/1.1933644

9. Yadollah F,Zabialah H,Fatemeh H,Fatemeh H, Orient J Chem., 2014, 30(2), 601-606; DOI: $10.13005 /$ ojc/300227

10. WBUNN C, Chemical crystallography. $2^{\text {nd }}$ Ed., Oxford Univ Press, London., 1961, p 220.

11. Kaufmann E N and Vianden R J, Rev Mod Phys., 1979, 51, 161-214.

12. Blaha P, Dufek P, Schwarz K and Haas H, Hyperfine Interactions., 1996, 97/98, 3.

13. Martinez-Pinedo G, Schwerdtfeger P, Caurier E, Langanke K, Nazarewicz W and Séihnel T, Phys Rev Lett., 2001, 87, 062701.

14. Cohen M H, Phys Rev., 1954, 96, 1278-1284.

15. Bersohn R, J Chem Phys., 1952, 20(10), 1505-1509; DOI:10.1063/1.1700203

16. Wang T C, Phys Rev., 1955, 99, 566-577.

17. Giavini T, Henrik B, Jørgen S and Hans J J, J Magn Reson., 2004, 166(2), 262-272; DOI:10.1016/j.jmr.2003.10.023 